\title{
Bouncing states of a droplet on a liquid surface under generalized forcing
}

\author{
R. Chacón, ${ }^{1,2}$ P. J. Martínez, ${ }^{3,4}$ and P.-M. Binder ${ }^{5}$ \\ ${ }^{1}$ Departamento de Física Aplicada, E.I.I., Universidad de Extremadura, Apartado Postal 382, E-06006 Badajoz, Spain \\ ${ }^{2}$ Instituto de Computación Científica Avanzada (ICCAEx), Universidad de Extremadura, E-06006 Badajoz, Spain \\ ${ }^{3}$ Departamento de Física Aplicada, E.I.N.A., Universidad de Zaragoza, E-50018 Zaragoza, Spain \\ ${ }^{4}$ Instituto de Ciencia de Materiales de Aragón, CSIC-Universidad de Zaragoza, E-50009 Zaragoza, Spain \\ ${ }^{5}$ Natural Sciences Division, University of Hawaii, Hilo, Hawaii 96720-4091, USA
}

(Received 29 May 2018; published 30 October 2018)

\begin{abstract}
Droplets can exhibit complex dynamics when vertically and sinusoidally forced by a liquid surface from which they remain separated by a thin air cushion. Here we extend previous studies to include a family of periodic forcing functions that vary smoothly from sinusoidal to square wave by changing a single parameter. Through analytical and numerical work we find that the dynamics of the droplets and transitions between regular and chaotic regimes are effectively controlled by the impulse imparted on the droplets over a half-period. We also find that having nonsinusoidal forcing lowers the threshold amplitudes for most of the dynamical regimes. This is explained on the basis of a correlation between impulse increases and subsequent energy increases.
\end{abstract}

DOI: 10.1103/PhysRevE.98.042215

\section{INTRODUCTION}

Since the pioneering work of Couder et al. [1] demonstrating that a droplet is able to bounce indefinitely without coalescing on the surface of a vertically vibrated liquid bath, the rebounding of such droplets has attracted a great deal of attention, partly because controlled droplet bouncing may be a promising way to manipulate fluids for microfluidic operations [2], and partly due to its reminiscence of the wave-particle duality $[3,4]$. The bouncing dynamics of drops atop a liquid bath vibrated sinusoidally at a given frequency has been previously studied [5-9], in the case where the vibration frequency corresponds to a given excited wavelength according to the dispersion relation of Faraday waves, while a simple theoretical model of the bouncing states has been developed $[8,10]$ for the bouncing dynamics on a soap film vertically and sinusoidally vibrated. Also, the vertical dynamics of a bouncing droplet on a bath subjected to forcing signals (FSs) composed of a combination of two commensurable frequencies has been experimentally studied in a recent work [11]. In all these cases diverse bouncing states were found, including periodic and multiperiodic states as well as chaos, as the amplitude of the harmonic FSs is varied. Beyond a critical amplitude threshold the stationary bouncing state becomes unstable due to the underlying wave field, giving rise to a walking regime in which the drops walk across the liquid surface.

In such previous works, however, the robustness of the bouncing scenario (BS) against diversity in the vertical vibrations of the liquid surface frame was not studied since harmonic vibrations have been systematically used because of their simplicity. Clearly, this mathematically convenient choice imposes a drastic and unnecessary restriction in the BS, limiting thus its possible implications and applications [12]. Thus, to fully explore and exploit the physics of the BS, it seems appropriate to consider FSs exhibiting general features of periodic signals which are the output of generic (nonlinear) systems, therefore being appropriately represented by Fourier series-not just by a single harmonic term. Since there are infinitely many different waveforms, an important question, both scientifically and technologically, is how can one explain in physical terms-providing in turn a quantitative characterization - the effect of the FS's waveform on the BS once its amplitude and period are fixed.

Here we theoretically and numerically show that for a generic FS $f(t)$ having equidistant zeros, the impulse transmitted by the signal over a half-period (hereafter referred to simply as the signal's impulse),

$$
I \equiv\left|\int_{0}^{T / 2} f(t) d t\right|
$$

is a relevant quantity that characterizes the effectiveness of such FS. Here $T$ is the period and $I$ a quantity integrating the conjoint effects of the signal's amplitude, period, and waveform. In the accelerating reference frame of the liquid surface, $f(t)$ introduces a fictitious inertial force $\ddot{f}(t)$ having the same period $T$ and an impulse given by

$$
I^{\prime} \equiv\left|\int_{0}^{T / 2} \ddot{f}(t) d t\right|=|\dot{f}(t=T / 2)-\dot{f}(t=0)|,
$$

i.e., $I^{\prime}$ is given by the (absolute) difference between the slopes of the FS at two of its consecutive zeros. The relevance of the signal's impulse has been observed previously in quite different physical contexts, such as adiabatically ac driven periodic Hamiltonian systems [13], chaotic dynamics of a pumpmodulation $\mathrm{Nd}: \mathrm{YVO}_{4}$ laser [14], ratchet transport [15-18], discrete breathers in nonlinear oscillator networks [19], topological amplification effects in scale-free networks of signaling devices [20], driven two-level systems and periodically curved waveguide arrays [21], and control of chaos in damped driven systems by secondary periodic excitations [22]. 


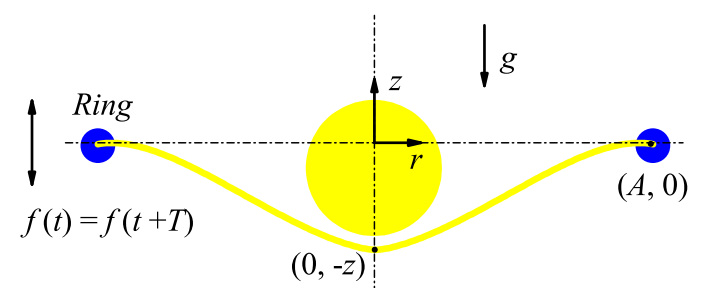

FIG. 1. Schematic representation of a droplet bouncing on a soap film vibrated with a vertical generic $T$-periodic displacement $f(t)$. The soap film is pinned at $(r, z)=(A, 0)$.

The effect of the FS's impulse will be studied below by generalizing the sinusoidal FS to a family of periodic functions, related to Jacobian elliptic functions, which vary smoothly from sinusoidal to square wave by varying a single parameter $(m)$. This family of signals will be introduced in Sec. II, along with a model that describes the behavior of droplets on a soap film in terms of a second-order ODE with two parameters, the previously introduced $m$ and an amplitude parameter, $\Gamma$. That section also describes the analytical and numerical tools used to address this problem, which include energy-based analysis, numerical integration, extraction of power spectra, and Lyapunov exponents (LEs) from the generated time series. Section III presents our results. We conclude the paper with Sec. IV, a general conclusion.

\section{MODEL SYSTEM AND FORCING FUNCTIONS}

Figure 1 shows a schematic representation of a droplet bouncing on a soap film vibrated with a vertical generic $T$ periodic displacement $f(t)$.

\section{A. Forcing functions}

Specifically, we study the effect of the FS's impulse by generalizing the sinusoidal FS to the case

$$
f(t)=\frac{B \arcsin [\sqrt{m} \operatorname{sn}(\Omega t ; m)]}{\arcsin (\sqrt{m})},
$$

where $B$ is the amplitude of the vertical vibrations of the accelerating frame, sn $(\cdot ; m)$ is the Jacobian elliptic function of parameter $m \in[0,1][K(m)$ is the complete elliptic integral of the first kind [23]], and $\Omega=\Omega(m, T) \equiv 4 K(m) / T$. When $m=0$, then $f(t)_{m=0}=B \sin (2 \pi t / T)$, i.e., one recovers the canonical case of a sinusoidal FS $[8,10]$, while in the other limit, $m=1$, one recovers a square wave function of amplitude $B$ and period $T$. The effect of renormalization of the elliptic sine argument in Eq. (3) is clear: with $T$ constant, solely the FS shape is varied by increasing the shape parameter $m$ from zero to 1 , and there is thus a smooth transition from a sine function to a square wave [see Fig. 2(a)].

Similar to the case of a sinusoidal FS, writing the force balance in the accelerating reference frame introduces the (dimensionless) periodic forcing term $-F(\tau)$ with

$$
F(\tau) \equiv \Gamma A \operatorname{sn}\left(\Omega^{\prime} \tau ; m\right) \operatorname{dn}\left(\Omega^{\prime} \tau ; m\right),
$$

where $\Gamma \equiv 4 \pi^{2} B /\left(g T^{\prime 2}\right), \quad A=A(m) \equiv 4 \sqrt{m} K^{2}(m) /\left[\pi^{2}\right.$ $\arcsin (\sqrt{m})], g$ is the gravitational acceleration, $\operatorname{dn}(\cdot ; m)$ is the Jacobian elliptic function of parameter $m \in[0,1], \Omega^{\prime}=$ $\Omega^{\prime}\left(m, T^{\prime}\right) \equiv 4 K(m) / T^{\prime}, T^{\prime} \equiv \sqrt{k / m_{d}} T$, and $\tau \equiv \sqrt{k / m_{d}} t$,
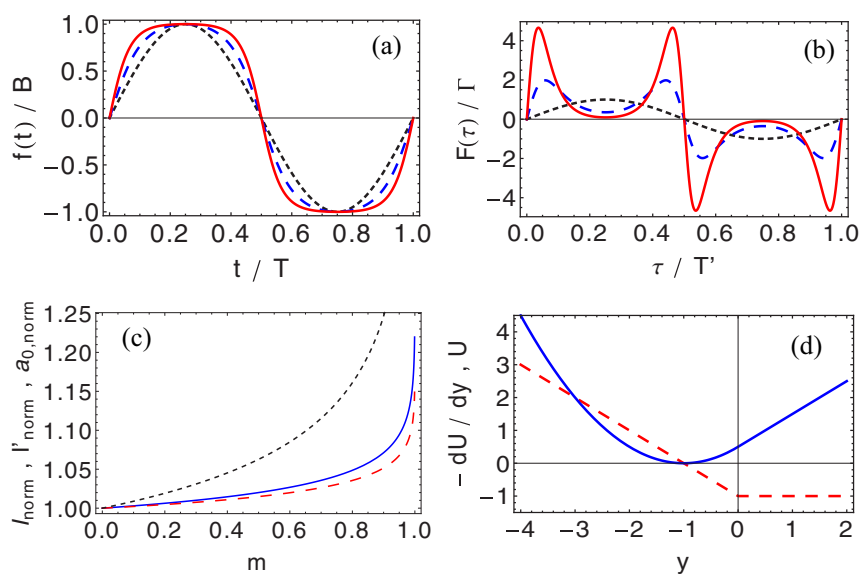

FIG. 2. (a) Forcing signal $f(t)$ [Eq. (3)] vs time over a signal period and (b) corresponding periodic forcing term in the accelerating reference frame $F(\tau)$ [Eq. (4)] vs time over a forcing period for three values of the shape parameter: $m=0,0.992$, and 0.999 (dotted, shaded, and solid lines, respectively). (c) Normalized impulse functions, $I_{\text {norm }} \equiv I(m) / I(m=0)$ [Eq. (5), solid line] and $I_{\text {norm }}^{\prime} \equiv$ $I^{\prime}(m) / I^{\prime}(m=0)$ [Eq. (6), dotted line], and normalized first Fourier coefficient of $f(t), a_{0, \text { norm }} \equiv a_{0}(m) / a_{0}(m=0)$ [Eq. (13), dashed line], vs the shape parameter $m$. (d) Effective force $-\frac{d U}{d y}(y) \equiv$ $-1-y H(-y)$ (dashed line) and corresponding effective potential $U(y) \equiv y+y^{2} H(-y) / 2+1 / 2$ associated with the forces exerted by the gravitational field and by the soap film.

with $k$ and $m_{d}$ being the effective spring constant [8] and the mass of the droplet, respectively. When $m=0$, then $F(\tau)_{m=0}=\Gamma \sin \left(\omega_{0} \tau\right)$ with $\omega_{0} \equiv \Omega^{\prime}\left(m=0, T^{\prime}\right)=2 \pi / T^{\prime}$, i.e., one recovers the case of a sinusoidal FS, while both the amplitude and the shape of $F(\tau)$ are varied by increasing the shape parameter $m$ from zero to 1 [see Fig. 1(b)]. After applying the definition given by Eq. (1) to the periodic functions $f(t)$ and $F(\tau)$ [cf. Eqs. (3) and (4), respectively] and using standard tables of integrals [24], one can obtain the dependence on the shape parameter $m$ of their respective impulses $I$ and $I^{\prime}$ [see Fig. 2(c)]:

$$
\begin{gathered}
I(m) \equiv \int_{0}^{T / 2} f(t) d t=B T \sum_{n=0}^{\infty} b_{n} a_{n}(m), \\
I^{\prime}(m) \equiv \int_{0}^{T^{\prime} / 2} F(\tau) d \tau=\frac{2 \Gamma T^{\prime} \sqrt{m} K(m)}{\pi^{2} \arcsin (\sqrt{m})},
\end{gathered}
$$

where the coefficients $b_{n}$ and $a_{n}(m)$ are given below. Note that the functions $I(m), I^{\prime}(m), a_{0}(m)$ monotonically increase as the shape parameter $m$ is increased from zero, while keeping constant $B$ and $T$, which allows us to expect impulse-induced dynamical changes in the BS, as shown below.

After Taylor expanding the function $\arcsin [\operatorname{sn}(\Omega t ; m)]$ in Eq. (3), one straightforwardly obtains the impulse $I(m)$ given by Eq. (5) with

$$
\begin{gathered}
b_{n} \equiv \frac{(2 n) !}{2^{2 n}(n !)^{2}(2 n+1)}, \\
a_{n}(m) \equiv \frac{m^{n+1 / 2}}{4 K(m) \arcsin (\sqrt{m})} \int_{0}^{2 K(m)} \operatorname{sn}^{2 n+1}(p ; m) d p,
\end{gathered}
$$


where the integrals in Eq. (8) can be obtained in closed analytical form by using Mathematica:

$$
\begin{gathered}
\int_{0}^{2 K(m)} \operatorname{sn}(p ; m) d p=\frac{2}{\sqrt{m}} \operatorname{arctanh}(\sqrt{m}), \\
\int_{0}^{2 K(m)} \operatorname{sn}^{3}(p ; m) d p=\frac{-\sqrt{m}+(1+m) \operatorname{arctanh}(\sqrt{m})}{m^{3 / 2}} \\
\int_{0}^{2 K(m)} \operatorname{sn}^{5}(p ; m) d p=\frac{-3 \sqrt{m}(1+m)}{4 m^{5 / 2}} \\
+\frac{[3+m(2+3 m)] \operatorname{arctanh}(\sqrt{m})}{4 m^{5 / 2}} \\
\cdots
\end{gathered}
$$

Similarly, after applying standard tables of integrals [24], one straightforwardly obtains the impulse $I^{\prime}(m)$ given by Eq. (6). We next provide the Fourier series of the FS $f(t)$ [Eq. (3)] and its second derivative $F(t) \equiv \ddot{f}(t)$ [Eq. (4)]. After noting that

$$
\dot{f}(t)=\frac{4 B \sqrt{m} K(m)}{T \arcsin (\sqrt{m})} \operatorname{cn}(\Omega t ; m)
$$

and using the Fourier series of the Jacobian elliptic function $[23] \mathrm{cn}(\cdot ; m)$, one obtains

$$
\begin{aligned}
f(t) & =B \sum_{n=0}^{\infty} a_{n}(m) \sin \left[(2 n+1) \Omega_{0} t\right], \\
a_{n}(m) & \equiv \frac{2 \operatorname{sech}\left[\left(n+\frac{1}{2}\right) \frac{\pi K(1-m)}{K(m)}\right]}{(2 n+1) \arcsin (\sqrt{m})},
\end{aligned}
$$

where $\Omega_{0} \equiv 2 \pi / T$. After taking the second derivative of the series in Eq. (13), one obtains

$$
\begin{gathered}
F(t)=g \Gamma \sum_{n=0}^{\infty} b_{n}(m) \sin \left[(2 n+1) \Omega_{0} t\right], \\
b_{n}(m) \equiv \frac{2(2 n+1) \operatorname{sech}\left[\left(n+\frac{1}{2}\right) \frac{\pi K(1-m)}{K(m)}\right]}{\arcsin (\sqrt{m})} .
\end{gathered}
$$

Note that $b_{n}(m)=(2 n+1)^{2} a_{n}(m)$, and hence

$$
\frac{b_{n}(m)}{b_{n}(m=0)}=\frac{a_{n}(m)}{a_{n}(m=0)}
$$

i.e., the dependence of the respective (normalized) Fourier coefficients of the FS and its second time derivative are the same, as expected, which allows us to understand the common dependence on the shape parameter of their associated (normalized) impulse functions [cf. Fig. 2(c)]. Figure 3 shows the relative size of the second and third Fourier coefficients with respect to the first one as a function of the shape parameter, illustrating thus the extremely rapid convergence of the Fourier series even for values of $m$ very close to 1 .

\section{B. Model system}

Introducing the $\mathrm{FS}$ just described into a model of bouncing states $[8,10]$ one obtains

$$
\begin{aligned}
\ddot{y}+d U / d y= & -H(-y) \Psi|\dot{y}| \dot{y}+\Gamma A \operatorname{sn}\left(\Omega^{\prime} \tau ; m\right) \\
& \times \operatorname{dn}\left(\Omega^{\prime} \tau ; m\right),
\end{aligned}
$$
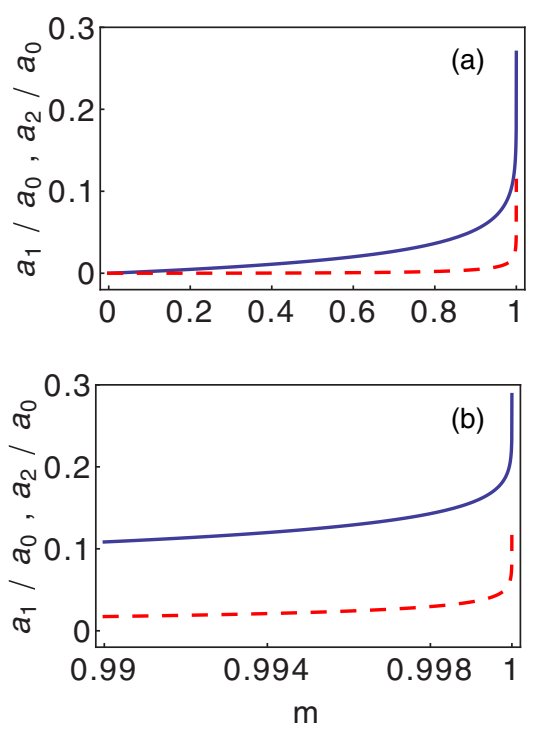

FIG. 3. Normalized second and third Fourier coefficients of the FS $f(t), a_{1}(m) / a_{0}(m)$ and $a_{2}(m) / a_{0}(m)$ [cf. Eq. (13); solid and dashed lines, respectively], vs the shape parameter $m$ over the ranges (a) $m \in\left[0,1-10^{-14}\right]$ and (b) $m \in\left[0.99,1-10^{-14}\right]$.

where all parameters and variables are dimensionless, $y \equiv$ $d y / d \tau, U(y) \equiv y+y^{2} H(-y) / 2+1 / 2$ is the effective potential associated with the forces exerted by the gravitational field and by the soap film [see Fig. 1(d)], $H(\cdot)$ is the Heaviside function, $\Psi$ is an experimentally adjustable parameter, while $y \equiv-k z /\left(m_{d} g\right)$ provides a measure of the vertical position of the droplets (see Fig. 1). This model will be analyzed in the next section.

\section{RESULTS}

\section{A. Numerical methods}

The differential system (16) was integrated by using a fourth-order Runge-Kutta method with time steps chosen in the range $\Delta \tau=0.001-0.005$. The simplest way to visualize the effect of the FS's impulse is to study the evolution of the attracting orbits as the shape parameter $m$ (or the normalized amplitude $\Gamma$ ) is varied. To provide different ways of dealing with the possibility of impulse-induced multiple attractors, we considered two options for the initial conditions for each of the parameter values of the bifurcation diagrams. In one case, we initialized on the last state found for the previous, slightly smaller parameter value (i.e., "following the attractor"), while we initialized on the same, fixed initial condition for each new parameter value in the other case. The numerical integration of Eq. (16) yields pseudo-orbits of the system in the form of time series $y(\tau), \dot{y}(\tau)$; a standard fast Fourier transform then yields the power spectrum $S(\omega)=|a(\omega)|^{2}$. Usual averaging procedures were used to improve its quality [25]. Examples of time series and corresponding power spectra are shown below. We calculated LEs by using a version of the algorithm introduced in Ref. [26], with integration typically up to $10^{4}$ drive cycles for each fixed set of parameters. To construct the LE diagrams we followed two steps. First, the maximal LE, $\lambda^{+}$, was calculated for each point on a $N \times N$ grid with 

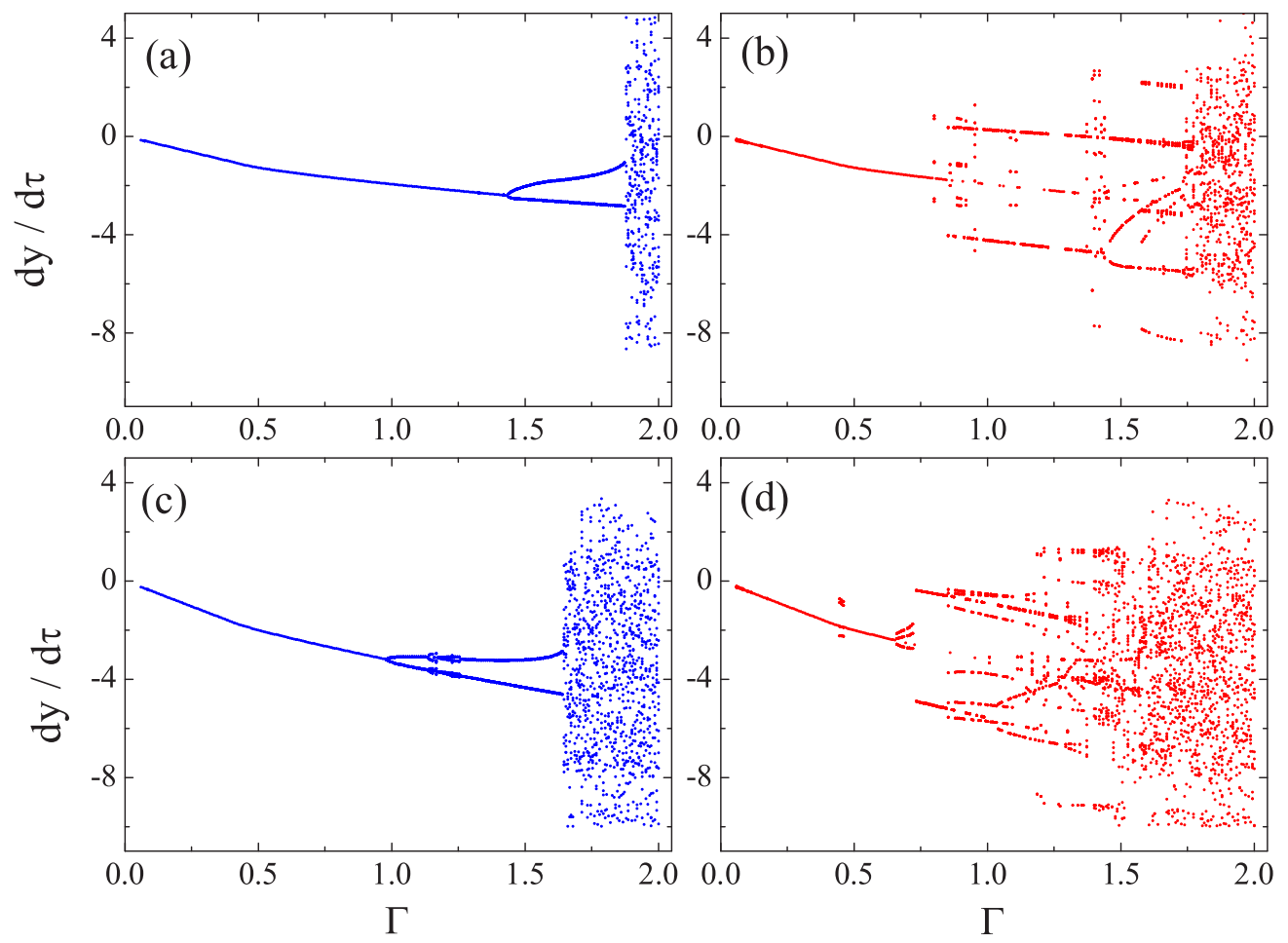

FIG. 4. Bifurcation diagrams for the (dimensionless) variable $d y / d \tau$ corresponding to Eq. (16), with the (dimensionless) amplitude factor $\Gamma$ in the range $0.06 \leq \Gamma \leq 2$ and two values of the shape parameter: (a), (b) $m=0$ and (c), (d) $m=0.9999$. The same initial conditions were set for every value of $\Gamma$ in versions (b) and (d), while versions (a) and (c) correspond to the case of initial conditions following the attractor. Fixed parameters: $\Psi=0.01, T^{\prime}=5.1927$.

amplitude factor $\Gamma$ and shape parameter $m$ along the horizontal and vertical axes. Second, a diagram was constructed by only plotting points on the grid according to a color code. The temporal average energy $\langle E\rangle$ was typically calculated over $10^{5}$ forcing cycles after the transients had been removed.

\section{B. Energy-based analysis}

By analyzing the variation of the droplets' energy, one can rationalize both the impulse-induced emergence of complexity (multiperiodicity) and the impulse-induced emergence of chaos. Indeed, Eq. (16) has the associated energy equation

$$
\frac{d E}{d \tau}=-H(-y) \Psi|\dot{y}| \dot{y}^{2}+\Gamma A \dot{y} \operatorname{sn}\left(\Omega^{\prime} \tau ; m\right) \operatorname{dn}\left(\Omega^{\prime} \tau ; m\right),
$$

where $E(\tau) \equiv \dot{y}^{2} / 2+U(y)$ is the energy function. Integration of Eq. (17) over any interval $\left[n T^{\prime}, n T^{\prime}+T^{\prime} / 2\right], n=$ $0,1,2, \ldots$, yields

$$
\begin{aligned}
& E\left(n T^{\prime}+T^{\prime} / 2\right) \\
& =E\left(n T^{\prime}\right)-\Psi \int_{n T^{\prime}}^{n T^{\prime}+T^{\prime} / 2} H(-y)|\dot{y}| \dot{y}^{2} d \tau \\
& \quad+\Gamma A \int_{n T^{\prime}}^{n T^{\prime}+T^{\prime} / 2} \dot{y} \operatorname{sn}\left(\Omega^{\prime} \tau ; m\right) \operatorname{dn}\left(\Omega^{\prime} \tau ; m\right) d \tau .
\end{aligned}
$$

Now, after applying the first mean value theorem for integrals [27] together with well-known properties of the Jacobian elliptic functions [23] to the last integral on the right-hand side of Eq. (18), one has

$$
\begin{aligned}
E\left(n T^{\prime}+T^{\prime} / 2\right)= & E\left(n T^{\prime}\right)-\Psi \int_{n T^{\prime}}^{n T^{\prime}+T^{\prime} / 2} H(-y)|\dot{y}| \dot{y}^{2} d \tau \\
& +\left.\Gamma T^{\prime} I^{\prime}(m)\right|_{\Gamma=T^{\prime}=1} \dot{y}\left(\tau^{*}\right)
\end{aligned}
$$

where the remaining integral in Eq. (19) represents the dissipation work, $\tau^{*} \in\left[n T^{\prime}, n T^{\prime}+T^{\prime} / 2\right]$, while

$$
\left.I^{\prime}(m)\right|_{\Gamma=T^{\prime}=1} \equiv \frac{2 \sqrt{m} K(m)}{\pi^{2} \arcsin (\sqrt{m})}
$$

is the impulse transmitted per unit of period and unit of amplitude [cf. Eq. (6)]. One can understand the occurrence of impulse-induced onset of chaos, by analyzing Eq. (19): a simple heuristic argument is given next. Let us consider fixing the parameters $\left(\Psi, \Gamma, T^{\prime}\right)$ for the droplet's motion to present a periodic orbit near the bottom of the potential well at $m=0$. Since the initial state is a steady (periodic) state, $\tau^{*}$ will depend solely on the FS but not on $n$. In this situation, one increases $m$ while holding the remaining parameters constant. For values $m>0$ such that the state of the bouncing droplet is still a periodic orbit (which will be relatively near the initial periodic orbit in phase space), one expects that $\dot{y}\left(\tau^{*}\right)$ and the remaining integral in Eq. (19) will maintain approximately their initial values (at $m=0$ ) while the impulse $\left.I^{\prime}(m)\right|_{\Gamma=T^{\prime}=1}$ will rise from its initial value. This means that, in some case depending upon the remaining parameters and the sign of $\dot{y}\left(\tau^{*}\right)$, the energy increment $\Delta E \equiv$ $E\left(n T^{\prime}+T^{\prime} / 2\right)-E\left(n T^{\prime}\right)>0$ could be enough to surpass the energy threshold to reach a different periodic attractor or a 

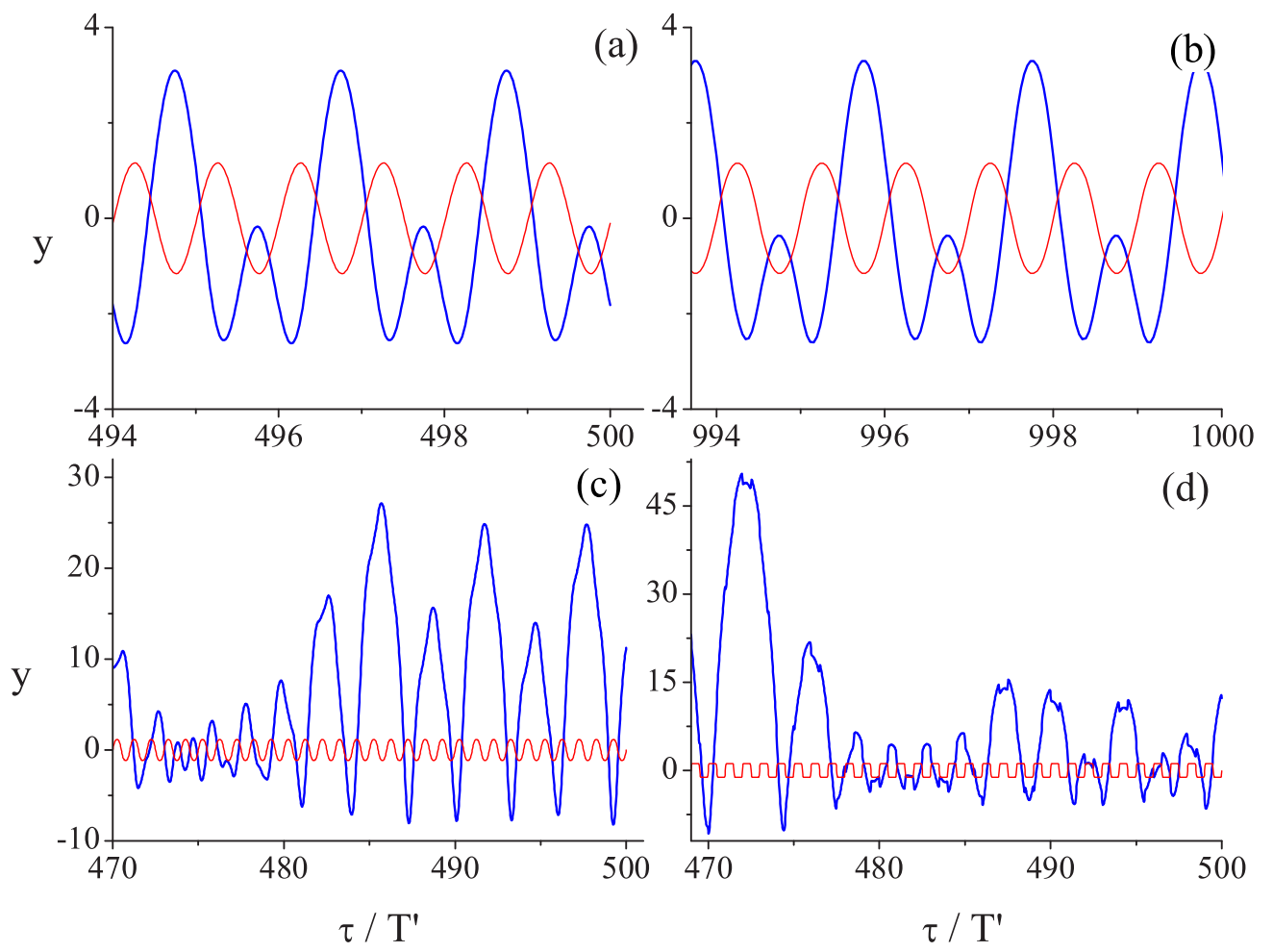

FIG. 5. Time series of the (dimensionless) droplet position $y$ [blue (black) thick line] and the forcing signal $f$ [red (gray) line; Eq. (3)] vs the number of driving cycles $\tau / T^{\prime} \equiv t / T$ for four values of the shape parameter: (a) $m=0$; (b) $m=0.78$; (c) $m=0.95$; (d) $m=1-10^{-14}$. Fixed parameters: $\Gamma=1.7, \Psi=0.01, T^{\prime}=5.1927$.

chaotic attractor, i.e., the oscillation amplitude threshold that will allow escape from the basin of attraction of the initial periodic orbit to be able to reach the basin of attraction of a different periodic orbit or that of a chaotic attractor. Clearly the probability of this event increases as the shape parameter $m$ is increased from zero, i.e., as the impulse transmitted is increased from the value corresponding to a sinusoidal FS.

\section{Bouncing scenario}

Figure 4 shows examples of bifurcation diagrams which were constructed by means of a Poincaré map at the parameters indicated in the caption to Fig. 4 and two values of the shape parameter: $m=0$ [(a) and (b)] and $m=0.9999$ [(c) and (d)]. Starting at $\Gamma=0.06$, and taking the transient time as 500 forcing periods after every increment of $\Delta \Gamma=(2 / 3) \times 10^{-2}$, we typically sampled 20 forcing periods by picking up the first $d y / d \tau$ value of every forcing cycle. The same initial conditions were set for every $\Gamma$ after $\Delta \Gamma$ was added in the diagrams shown in panels (b) and (d), while those shown in panels (a) and (c) correspond to the case of initial conditions following the attractor. Examples of time series $y(\tau)$ for several values of the shape parameter and the corresponding power spectra are shown in Figs. 5 and 6. When the FS is sinusoidal $(m=0)$ one has a period-2 solution [Figs. 5(a) and 6]. After slightly increasing the impulse $(m=0.78)$, a period-2 solution still occurs [Fig. 5(b)], such that only the peaks corresponding to odd subharmonics, $\omega=3 \omega_{0}, 5 \omega_{0}, 7 \omega_{0}, \ldots$, are higher than those associated with the power spectrum of the period-2 solution existing for $m=0$ (compare the two lowermost lines in Fig. 6). Further increase of the impulse may give rise to chaos, as for $m=0.95$ [cf. Fig. 5(c)], which is reflected in the visible enhancement of the power background in the corresponding power spectrum (second line from the top in Fig. 6). This further effect is ever higher as $m \rightarrow 1$, i.e., as the FS approximates a square wave function [see Fig. 2(a)], as for

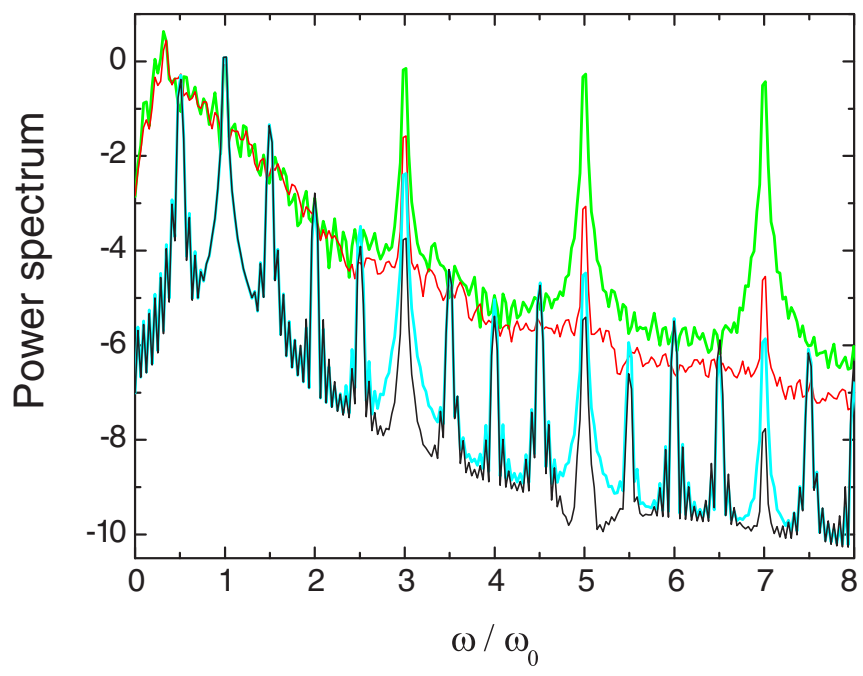

FIG. 6. Power spectra $\left[\log _{10}|S(\omega)|\right.$ vs $\omega / \omega_{0}$, with $\left.\omega_{0} \equiv 2 \pi / T^{\prime}\right]$ corresponding to the time series $\dot{y}(\tau)$ arising from Eq. (16) for four values of the shape parameter: $m=0,0.78,0.95$, and $1-10^{-14}$ (lines from bottom to top, respectively). Fixed parameters: $\Gamma=$ 1.7, $\Psi=0.01, T^{\prime}=5.1927$. 
$m=1-10^{-14}$ [Fig. 5(d) and uppermost line in Fig. 6]. The power of the four spectra shown in Fig. 6 is exactly the same at the fundamental (driving) frequency $\omega=\omega_{0} \equiv 2 \pi / T^{\prime}$, as expected.

As already mentioned above, Fig. 3 shows the relative dependence on the shape parameter of the second, $a_{1}(m)$, and third, $a_{2}(m)$, Fourier coefficients of the FS [cf. Eqs. (13) and (14)] with respect to the first one, $a_{0}(m)$, indicating the extremely rapid convergence of the Fourier series even for values of $m$ very close to 1 . Thus, regarding experiments, this property is relevant in the sense that it allows one to effectively approximate the elliptic FS by solely retaining the first two harmonics of its Fourier expansion over a sufficiently large range of values of the shape parameter $(0 \leq m \lesssim 0.99$; see Fig. 3):

$$
f(t) \approx B\left[a_{0}(m) \sin \left(\Omega_{0} t\right)+a_{1}(m) \sin \left(3 \Omega_{0} t\right)\right],
$$

where $\Omega_{0} \equiv 2 \pi / T$ [cf. Eq. (13)]. The second-order nonautonomous differential equation (16) may be solved subject to the initial conditions $y(0)=0, \dot{y}(0)=-V$ at impact. Here we are mainly interested in investigating the structural stability of the BS under changes in the FS's impulse (i.e., in the shape parameter $m$ ), while keeping constant the remaining parameters. For the sake of comparison with previous studies, we will assume parameter values which were used in the simple case of a soap film vertically and sinusoidally vibrated $[8,10]$. Our numerical simulations show that an effect of increasing the impulse transmitted by the FS is to diminish the value of its amplitude factor $\Gamma$ at which the bouncing droplet undergoes a period-doubling transition both in the case of initial conditions "following the attractor" and in the case of fixed initial conditions (see Fig. 4). Another effect of increasing the impulse is to diminish the threshold value of the amplitude factor $\Gamma$ at which the chaotic behavior appears, $\Gamma_{\text {chaos }}=\Gamma_{\text {chaos }}(m)$, again in the two cases of initial conditions (see Fig. 4). The same aforementioned impulse-induced dynamical features can be seen in the bifurcation diagrams for the variable $d y / d \tau$ with the shape parameter $m$ in the range $0 \leq m \leq 1-10^{-14}$, which are shown in Fig. 7 .

Additional information of the bouncing motions appearing at different values of the shape parameter can be obtained by analyzing the spectral properties of the corresponding solutions (see Fig. 6). The comparison of such power spectra indicates that only the power associated with the peaks corresponding to odd subharmonics increases as the impulse transmitted is increased (i.e., as $m$ is increased from zero), which is a consequence of the increase of the relative weight of such subharmonics in the Fourier series of both $f(t)$ and $F(\tau)$ [Eqs. (13) and (14), respectively] as $m \rightarrow 1$. Furthermore, our numerical simulations indicate that the extension of the region in phase space which is visited by the corresponding chaotic attractors existing at $\Gamma>\Gamma_{\text {chaos }}(m)$ increases as the shape parameter $m$ is increased, i.e., as the impulse transmitted is increased. We systematically studied the chaotic regime by calculating Lyapunov exponents (LEs), as in the example shown in Fig. 8. One sees again that the effect of increasing the impulse (i.e., the shape parameter $m$ ) is to diminish the threshold value of the amplitude factor $\Gamma$ at which the
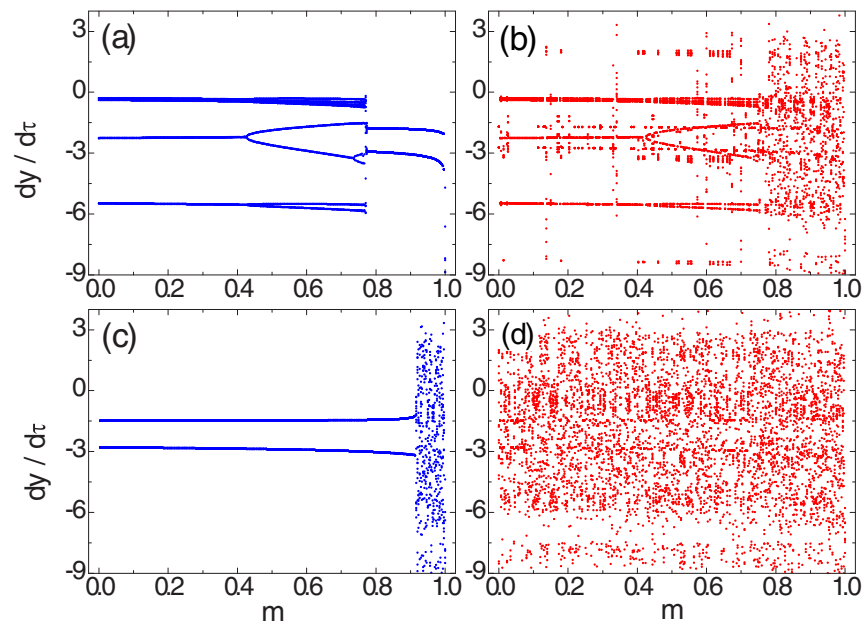

FIG. 7. Bifurcation diagrams for the (dimensionless) variable $d y / d \tau$ with the (dimensionless) shape parameter $m$ in the range $0 \leqslant m \leqslant 1-10^{-14}$ and two values of the amplitude factor: (a), (b) $\Gamma=1.7$ and (c), (d) $\Gamma=1.8$. The same initial conditions were set for every value of $m$ in versions (b) and (d), while versions (a) and (c) correspond to the case of initial conditions following the attractor. Fixed parameters: $\Psi=0.01, T^{\prime}=5.1927$.

chaotic behavior appears: $\Gamma_{\text {chaos }}\left(m>m^{\star}\right)<\Gamma_{\text {chaos }}\left(m^{\star}\right)$ with $0 \leqslant m^{\star}<1$.

Remarkably, our computations of the temporal average energy, $\langle E\rangle$, confirmed the predictions from the energy-based analysis (see Sec. III B). Indeed, we found an excellent correlation among the temporal average energy $\langle E\rangle$, the impulse $I^{\prime}$ [Eq. (6)], and the maximal LE $\lambda^{+}$as functions of the shape parameter $m$ over the complete range of periodic solutions (compare Figs. 2 and 9), while we found an approximate correlation over the complete range of chaotic solutions (see Fig. 9, bottom panels). The asymptotic behavior of $\langle E\rangle$ and $\lambda^{+}$as $m \rightarrow 1$ is ultimately due to the functional dependence [24] of $K(m)$ on $m$.

When the coefficient $\Psi$ [Eq. (16)] is sufficiently small, one can expect the appearance of the phenomenon of

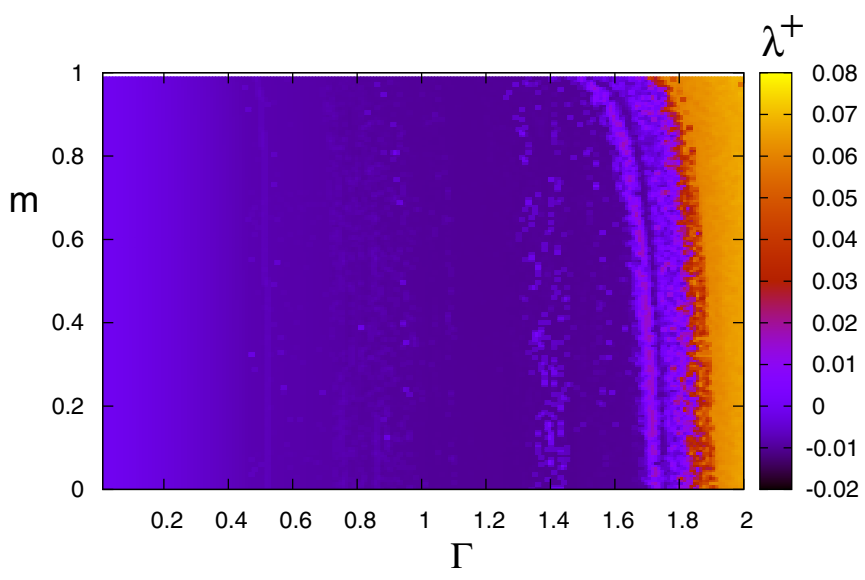

FIG. 8. Maximal Lyapunov exponent $\left(\lambda^{+}\right)$distribution in the $(\Gamma-m)$ parameter plane corresponding to solutions of the model (16) for $\Psi=0.01, T^{\prime}=5.1927$. 

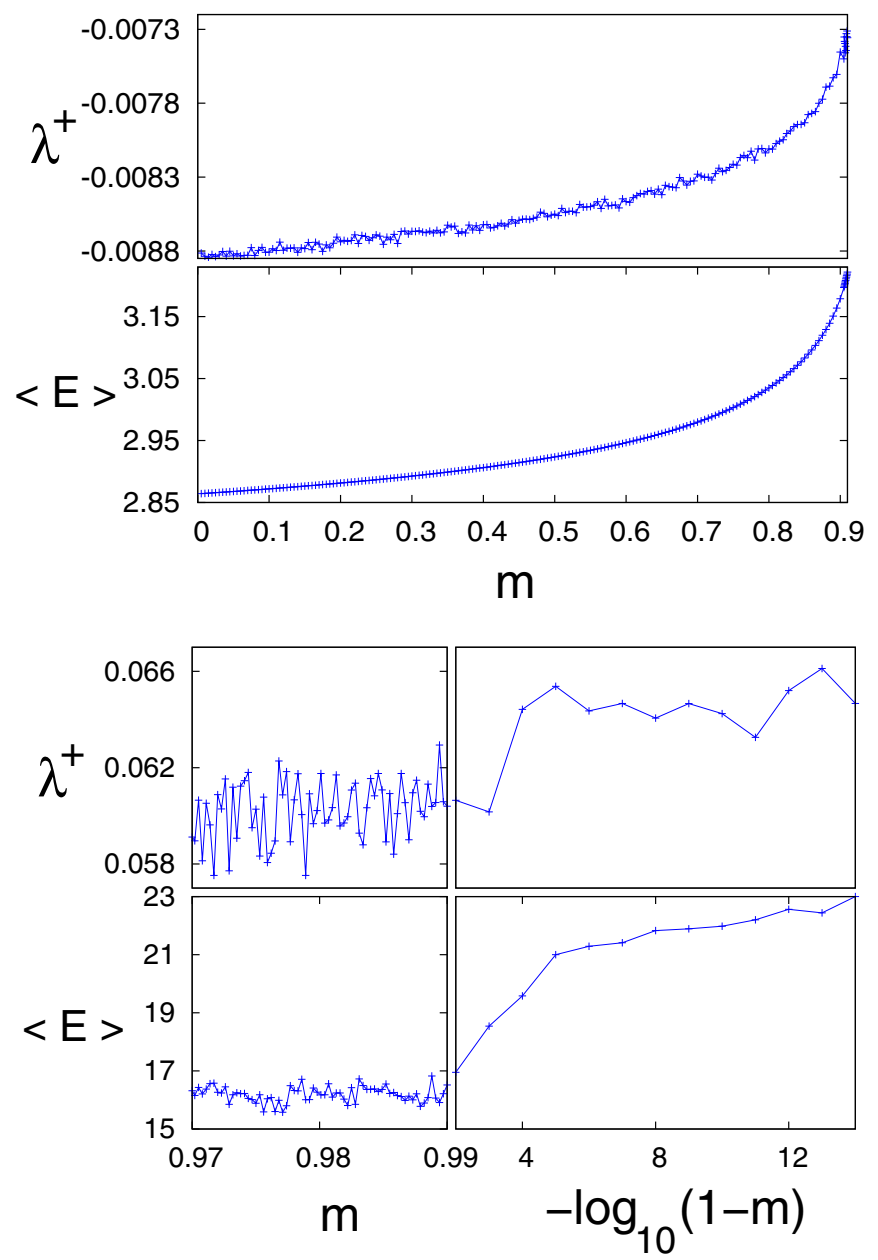

FIG. 9. Maximal LE, $\lambda^{+}$, and temporal average energy, $\langle E\rangle$, as functions of the shape parameter $m$ over (top panels) periodic and (bottom panels) chaotic ranges for $\Psi=0.01, \Gamma=1.8, T^{\prime}=$ 5.1927. Solid lines are solely plotted to guide the eye.

multistability [28]. We have indeed detected the coexistence of different attractors when the amplitude parameter $\Gamma$ is varied for a given set of the remaining parameters. Figure 10 shows an example for four randomly chosen initial conditions and $m=0, \Psi=0.01$. We found that multistability occurs over an intermediate range of $\Gamma$ values between two ranges of monostability (a period-1 attractor for sufficiently small amplitudes and a chaotic attractor for sufficiently large amplitudes). Remarkably, we also observed multistability when the shape parameter $m$ is varied while the remaining parameters are held constant. Figure 11 shows an example for four randomly chosen initial conditions and $\Gamma=0.04, \Psi=0.01$. One sees the coexistence of period-1, period-2, and period-3 attractors over distinct ranges of $m$ values. Thus reshaping a FS appears as an alternative procedure to control multistability [28].

\section{CONCLUSIONS}

Our theoretical and numerical results show that the impulse transmitted by the forcing signal which yields vertical vibrations of a liquid surface frame is a fundamental quantity

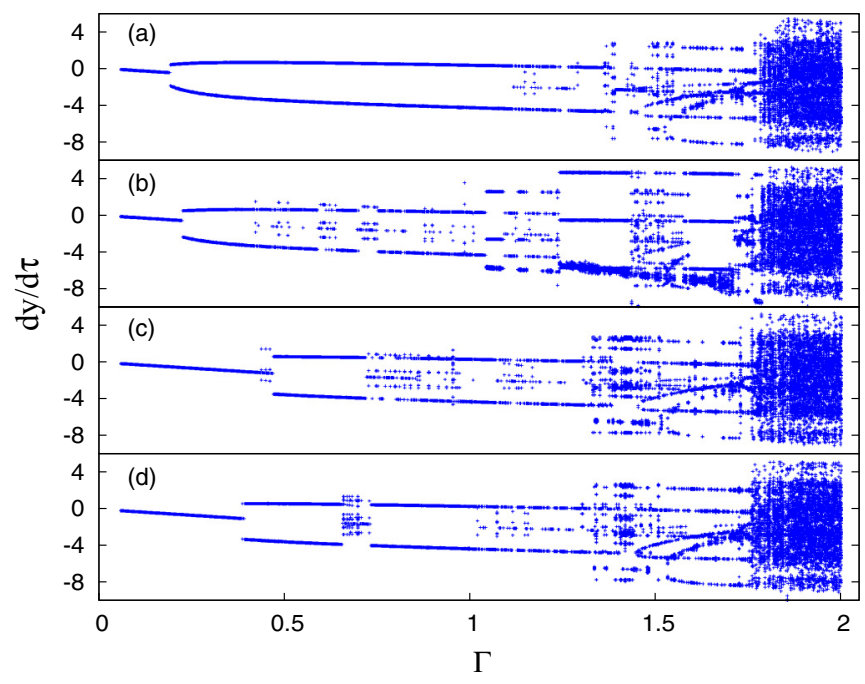

FIG. 10. Bifurcation diagrams for the (dimensionless) variable $d y / d \tau$ corresponding to Eq. (16), with the (dimensionless) amplitude factor $\Gamma$ in the range $0.06 \leqslant \Gamma \leqslant 2$ and four randomly chosen initial conditions in versions (a), (b), (c), and (d), respectively. The same initial conditions were set for every value of $\Gamma$ in each version. Fixed parameters: $\Psi=0.01, T^{\prime}=5.1927, m=0$.

for the reliable control of the scenario associated with the stationary bouncing regime of droplets. Specifically, we have shown through a single model that the values of the forcing signal's amplitude at which different dynamical bifurcations occur, such as transitions from simple periodic states to complex periodic, as well as transitions from periodic states to chaotic states can be suitably diminished by increasing the impulse transmitted, while keeping constant the remaining parameters. We have analytically shown that this effect of the impulse may be understood on the basis of a correlation

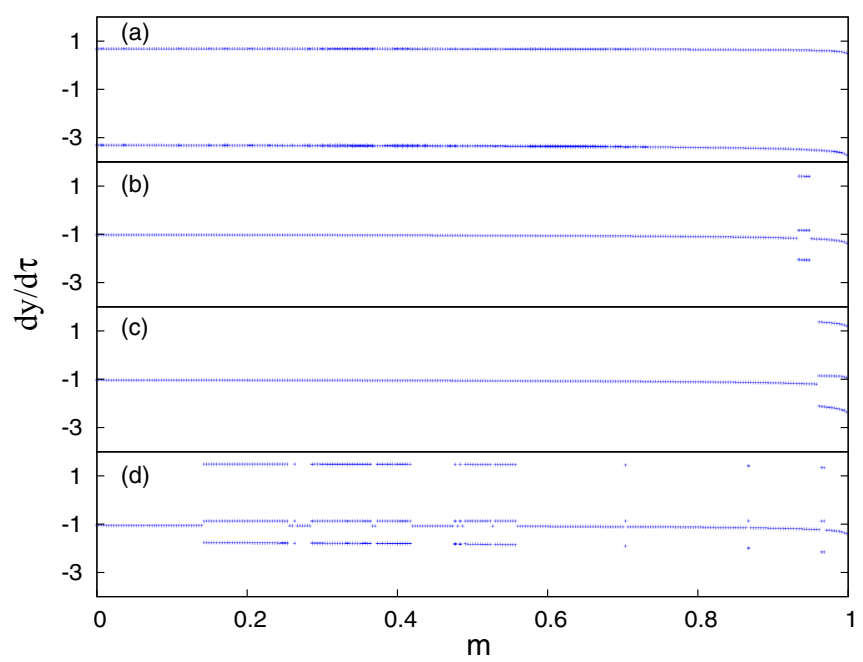

FIG. 11. Bifurcation diagrams for the (dimensionless) variable $d y / d \tau$ with the (dimensionless) shape parameter $m$ in the range $0 \leqslant$ $m \leqslant 0.999$ and four randomly chosen initial conditions in versions (a), (b), (c), and (d), respectively. The same initial conditions were set for every value of $m$ in each version. Fixed parameters: $\Psi=0.01$, $T^{\prime}=5.1927, \Gamma=0.4$. 
between increases of the impulse and subsequent increases of the energy transmitted by the vertical acceleration of the liquid surface. We have also found that multistability can be effectively controlled by the impulse imparted on the droplets.

Moreover, the present bouncing droplets scenario contains and extends the prevalent one, since the latter essentially arises from the systematic application of sinusoidal forcing signals and hence its control depends upon two quantities that have systematically been assumed to be independent of each other: the amplitude and the frequency of the sinusoidal signal. In contrast, the present bouncing droplets scenario identifies a single quantity - the impulse transmitted by the forcing signal per unit of period and unit of amplitudewhich is controlled by a single parameter while providing reliable control of the droplets' dynamic irrespective of the forcing signal waveform. It is worth mentioning that two FSs transmitting the same impulse but with different period and amplitude can yield different BSs because of the possible existence of resonances with underlying periodic solutions.
More importantly, we have shown that an effective experimental realization of the impulse-based control of the droplets' dynamic can be readily achieved by solely implementing the two first harmonics of the Fourier series of our generalized forcing signal, which facilitates its application in microfluidics. In addition, our work could shed some light on our understanding and control of the hydrodynamics of pilot wave systems.

\section{ACKNOWLEDGMENTS}

R.C. gratefully acknowledges financial support from the Junta de Extremadura (JEx, Spain) through Project No. GR18081 cofinanced by FEDER funds. P.J.M. acknowledges financial support from the Ministerio de Economía y Competitividad (MINECO, Spain) through Project No. FIS201787519 cofinanced by FEDER funds and from the Gobierno de Aragón (DGA, Spain) through Grant E36_17R to the FENOL group.
[1] Y. Couder, E. Fort, C. H. Gautier, and A. Boudaoud, Phys. Rev. Lett. 94, 177801 (2005).

[2] M. Brandenbourger, N. Vandewalle, and S. Dorbolo, Phys. Rev. Lett. 116, 044501 (2016).

[3] J. W. M. Bush, Annu. Rev. Fluid Mech. 47, 269 (2015).

[4] J. W. M. Bush, Phys. Today 68(8), 47 (2015).

[5] A. Eddi, E. Sultan, J. Moukhtar, E. Fort, M. Rossi, and Y. Couder, J. Fluid Mech. 674, 433 (2011).

[6] S. Protiere, A. Boudaoud, and Y. Couder, J. Fluid Mech. 554, 85 (2006).

[7] S. Dorbolo, D. Terwagne, N. Vandewalle, and T. Gilet, New J. Phys. 10, 113021 (2008).

[8] T. Gilet and J. W. M. Bush, J. Fluid Mech. 625, 167 (2009).

[9] J. Molacek and J. W. M. Bush, J. Fluid Mech. 727, 582 (2013).

[10] T. Gilet and J. W. M. Bush, Phys. Rev. Lett. 102, 014501 (2009); 103, 019901(E) (2009).

[11] N. Sampara and T. Gilet, Phys. Rev. E 94, 053112 (2016).

[12] P. A. Milewski, C. A. Galeano-Rios, A. Nachbin, and J. W. M. Bush, J. Fluid Mech. 778, 361 (2015).

[13] R. Chacón, M. Yu. Uleysky, and D. Makarov, Europhys. Lett. 90, 40003 (2010).

[14] M.-D. Wei and C.-C. Hsu, Opt. Commun. 285, 1366 (2012).

[15] R. Chacón, J. Phys. A 40, F413 (2007).
[16] R. Chacón, J. Phys. A 43, 322001 (2010).

[17] P. J. Martínez and R. Chacón, Phys. Rev. Lett. 100, 144101 (2008).

[18] M. Rietmann, R. Carretero-González, and R. Chacón, Phys. Rev. A 83, 053617 (2011).

[19] J. Cuevas-Maraver, R. Chacón, and F. Palmero, Phys. Rev. E 94, 062206 (2016).

[20] P. J. Martínez and R. Chacón, Phys. Rev. E 93, 042311 (2016).

[21] R. Chacón, Phys. Rev. A 85, 013813 (2012).

[22] P. J. Martínez, S. Euzzor, J. A. C. Gallas, R. Meucci, and R. Chacón, Sci. Rep. 7, 17988 (2017).

[23] J. V. Armitage and W. F. Eberlein, Elliptic Functions (Cambridge University Press, Cambridge, UK, 2006).

[24] P. F. Byrd and M. D. Friedman, Handbook of Elliptic Integrals for Engineers and Scientists (Springer-Verlag, Berlin, 1971).

[25] W. H. Press, B. P. Flannery, S. A. Teukolsky, and W. T. Vetterling, Numerical Recipes. The Art of Scientific Computing (Cambridge University Press, Cambridge, UK, 1988).

[26] A. Wolf, J. B. Swift, H. L. Swinney, and J. A. Vastano, Physica D 16, 285 (1985).

[27] I. S. Gradshteyn and I. M. Rizhik, Table of Integrals, Series, and Products (Academic Press, New York, 1980).

[28] A. N. Pisarchik and U. Fendel, Phys. Rep. 540, 167 (2014) 